\title{
Pioglitazone Therapy Increases Insulin-Stimulated Release of D-Chiro-Inositol-Containing Inositolphosphoglycan Mediator in Women with Polycystic Ovary Syndrome
}

\author{
Anshu Gupta, MD, MS, Daniela Jakubowicz, MD, ${ }^{2,3}$ and John E. Nestler, MD ${ }^{4}$
}

\begin{abstract}
Background: Insulin resistance in women with polycystic ovary syndrome (PCOS) may be mediated, in part, by a deficiency in the insulin-stimulated release of a D-chiro-inositol-inositolphosphoglycan (DCI-IPG) mediator of insulin action. Supporting this idea, several studies have reported improved insulin sensitivity in both lean and obese women with PCOS after oral administration of DCI. Pioglitazone improves insulin sensitivity in women with PCOS, but it is unknown whether this may be contributed by enhanced insulin-stimulated release of the DCI-IPG second messenger. The study aimed to determine if pioglitazone increases release of biologically active DCI-IPG per unit insulin released during an oral glucose tolerance test (OGTT).

Methods: A randomized, double-blind placebo-controlled trial was conducted in 32 women with PCOS at a tertiary referral center in Venezuela. The intervention comprised administration of pioglitazone $45 \mathrm{mg}$ daily or

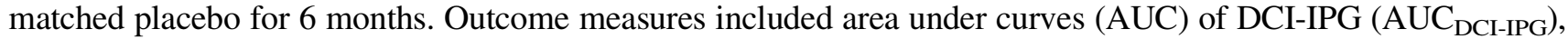
insulin $\left(\mathrm{AUC}_{\text {insulin }}\right)$, and the ratio of $\mathrm{AUC}_{\mathrm{DCI}-\mathrm{IPG}}$ to $\mathrm{AUC}_{\text {insulin }}$ during a 2-hr OGTT.

Results: After treatment with pioglitazone, $\mathrm{AUC}_{\text {insulin }}$ during the OGTT decreased and whole body insulin sensitivity, as determined by the Matsuda index, increased significantly only in the pioglitazone group. The ratio of $\mathrm{AUC}_{\mathrm{DCI}-\mathrm{IPG}} / \mathrm{AUC}_{\mathrm{insulin}}$ increased in the pioglitazone group by 1.85 -fold $(P<0.0001)$ with no significant change in the placebo group. Change in Matsuda index correlated with change in DCI-IPG released per unit of insulin during OGTT $(r=0.47, P<0.01)$.

Conclusion: In women with PCOS, pioglitazone increased insulin-stimulated release of the DCI-IPG second messenger of insulin action, which may contribute to its insulin-sensitizing effect in these women.
\end{abstract}

\section{Introduction}

$\mathrm{P}$ OLYCYSTIC OVARY SYNDROME (PCOS) is a common disorder affecting $6 \%-10 \%$ of reproductive age women ${ }^{1,2}$ with significant implications for fertility; it is also associated with co-morbidities such as hypertension, diabetes, dyslipidemia, and cardiovascular disease. Numerous studies have established a central role of insulin resistance (IR) and compensatory hyperinsulinemia in the pathogenesis of the hyperandrogenism and chronic anovulation of PCOS. ${ }^{3,4}$ Multiple lines of evidence suggest that a deficiency of a putative inositolphosphoglycan (IPG) second messenger that mediates insulin action, ${ }^{5}$ specifically the D-chiro-inositol- inositolphosphoglycan mediator (DCI-IPG), contributes to IR in both individuals with type 2 diabetes (T2DM) and in women with PCOS. ${ }^{6,7}$ Insulin-stimulated release of DCIIPG mediator correlates with insulin sensitivity in women with PCOS. ${ }^{8}$ Furthermore, insulin action is coupled to release of DCI-IPG, an insulinomimetic second messenger that promotes glycogen synthesis. ${ }^{9}$ Recently, it was reported that the coupling between insulin action and the release of the DCI-IPG mediator is selectively impaired in PCOS women with obesity this suggests that insulin-stimulated release of the DCI-IPG mediator is defective in obese women with PCOS. ${ }^{10}$ Moreover, interventional studies have reported that oral administration of DCI reduced circulating insulin,

\footnotetext{
${ }^{1}$ Department of Pediatrics, Virginia Commonwealth University, Richmond, Virginia.

${ }^{2}$ Department of Internal Medicine, Obesity, Diabetes and Fertility Unit, Hospital de Clinicas Caracas, Central University of Venezuela, Caracas, Venezuela.

${ }^{3}$ Diabetes Unit, Wolfson Medical Center, Sackler Faculty of Medicine, Tel Aviv University, Holon, Israel.

${ }^{4}$ Department of Internal Medicine, Virginia Commonwealth University, Richmond, Virginia.
} 
decreased serum androgens, and improved ovulatory frequency in obese women with PCOS. ${ }^{1-14}$

We previously reported that metformin increased the release of bioactive DCI-IPG per unit of insulin released during an oral glucose challenge, ${ }^{6}$ further supporting the contribution of DCI-IPG deficiency to the IR in these women. Pioglitazone is a PPAR- $\gamma$ (peroxisome proliferator-activated receptor gamma, also known as the glitazone receptor) or NR1C3 (nuclear receptor subfamily 1, group C, member 3) receptor agonist that enhances insulin sensitivity and has been demonstrated to improve ovulatory function and lower serum androgen levels in women with PCOS. ${ }^{13}$ However, the effect of pioglitazone on the insulin-stimulated release of the DCIIPG mediator is not known. We hypothesized that, in a manner similar to that reported for metformin, ${ }^{6}$ pioglitazone improves insulin action in women with PCOS, in part, by increasing insulin-stimulated release of DCI-IPG. To test this hypothesis, we conducted a double-blind placebo-controlled study, in which we assessed the release of both insulin and DCI-IPG during an oral glucose tolerance test (OGTT) at baseline and after 6 months of treatment with either placebo or pioglitazone.

\section{Materials and Methods}

The study was conducted at Virginia Commonwealth University (VCU), Richmond, VA (A.G., J.E.N.), and the Hospital de Clincas Caracas, Caracas, Venezuela (D.J.), as a randomized, double-blind placebo-controlled trial, in which insulin-stimulated release of the DCI-IPG mediator in response to glucose during a standard OGTT was assessed at baseline and after treatment with pioglitazone or matched placebo for 6 months in women with PCOS. A total of 32 [body mass index (BMI) $\geq 24 \mathrm{~kg} / \mathrm{m}^{2}$ ] women with PCOS, between 18 and 40 years old, were studied during the equivalent of the follicular phase of the menstrual cycle, as documented by serum progesterone of $\leq 2 \mathrm{ng} / \mathrm{mL}$.

PCOS was defined using the 1990 NIH/NICHD criteria ${ }^{15}$; that is, all PCOS women had chronic oligomenorrhea (eight or fewer menstrual periods annually) and biochemical hyperandrogenemia (elevated serum-free testosterone), and secondary causes of hyperandrogenism or ovulatory dysfunction were excluded by a normal thyroid stimulating hormone, prolactin, and $17 \alpha$-hydroxyprogesterone $<200 \mathrm{ng} / \mathrm{dL}$. All women underwent screening with a 2-hour $75 \mathrm{~g}$ dextrose OGTT to exclude diabetes mellitus. Women who had disorders associated with IR, such as hypertension or dyslipidemia, were not excluded as long as they had been on a stable dose of medication for 6 months. Exclusion criteria included the following: (1) diabetes mellitus by fasting glucose or OGTT, or clinically significant pulmonary, cardiac, renal, hepatic, neurologic, psychiatric, infectious, neoplastic, and malignant disease (other than nonmelanoma skin cancer); (2) current use of oral contraceptives, steroids, or anorectic drugs; (3) documented or suspected recent (within 1 year) history of drug abuse or alcoholism; or (4) ingestion of any investigational drug or being on a weight loss diet within 2 months before study onset. The study was approved by the Institutional Review Board of Virginia Commonwealth University, and signed informed consent was obtained from all participants.

Because DCI may be taken in as part of a diet high in legumes or fruits, all participants were interviewed by a dietitian to identify those who may be consuming diets containing unusually high amounts of inositols. The women were given instructions for a balanced mixed diet to be followed for at least 3 days before the start of the study.

On day 1 , the participants came to the Hospital de Clinicas Caracas at 08:00 h after a 12-h overnight fast, and fasting blood samples were drawn at 08:00, 08:15, and $08: 30 \mathrm{~h}$ and pooled for determination of liver function tests and plasma insulin, glucose, and sex steroids (testosterone). At 09:00 h, an OGTT was performed by administering $75 \mathrm{~g}$ dextrose orally and collecting blood samples for determination of serum glucose and insulin every $60 \mathrm{~min}$, and DCIIPG every $15 \mathrm{~min}$ for $2 \mathrm{hr}$. Height and weight were measured to the nearest $0.1 \mathrm{~cm}$ and $0.1 \mathrm{~kg}$ using a precision stadiometer and digital scale, respectively. Waist was measured at the level of the umbilicus, and hip circumference was measured at the widest diameter of the buttocks to the nearest $0.1 \mathrm{~cm}$. Waist/hip ratio was calculated subsequently.

After completing the OGTT, women were randomized to receive either pioglitazone $45 \mathrm{mg}$ or matched placebo once daily for 6 months. This dose and duration of pioglitazone administration have been previously demonstrated to markedly improve insulin sensitivity and reduce circulating insulin in women with $\operatorname{PCOS}^{16}$ and is well tolerated. The women were allowed to eat ad libitum while an outpatient, but were instructed to not alter their eating habits, activity level, or lifestyle during the study. The women also maintained a daily diary of menstrual bleeding.

The women returned to research clinic every 4 weeks for monitoring. After having taken pioglitazone for 6 months, they returned on day 164 to confirm that they were in the equivalent of the follicular phase of the menstrual cycle by a serum progesterone level. On day 166, all tests obtained on day 1 were repeated. Since pioglitazone may improve ovulation frequency in some women, if a woman was found to be in the luteal phase of the menstrual cycle on day 164, she was continued on pioglitazone for an additional 2-3 weeks and then studied when she was documented to be in the equivalent of the follicular phase.

Only women with normal hepatic and cardiac function were studied. Liver function tests were monitored at 4, 12, and 20 weeks of the study. To assess compliance with study medication (pioglitazone or placebo), the women returned to the Hospital de Clinicas Caracas on a monthly basis and a capsule count was performed. Participants were considered compliant if they had taken more than $80 \%$ or more of all drugs (pioglitazone or placebo) at each visit based on audit of returned/dispensed capsules.

Pioglitazone and matched placebo were provided by Takeda Pharmaceuticals; Takeda did not provide any other support for the study. All glucose and insulin samples were centrifuged immediately, and sera were stored at $-20^{\circ} \mathrm{C}$ until assayed. Glucose, insulin, and free testosterone were analyzed in the clinical laboratory of the Hospital de Clinicas Caracas. DCI-IPG bioactivity was measured using an in-house bioactivity assay developed by the laboratory of J.E.N., as previously described. ${ }^{6}$

\section{Data analysis}

All data for participants were analyzed as assigned to their respective groups at randomization. We examined the response of serum insulin concentrations and the relative bioactivity of DCI-IPG to the oral administration of glucose 
by calculating the areas under the respective response curves (AUCs) by the trapezoidal rule. Since insulin is thought to mediate the release of DCI-IPG after a glucose load ${ }^{17}$ and there are interparticipant variations in $\mathrm{AUC}_{\text {insulin, the ratio }}$ of $\mathrm{AUC}_{\mathrm{DCI}-\mathrm{IPG}} / \mathrm{AUC}_{\text {insulin }}$ more accurately reflects insulinmediated release of DCI-IPG than $\mathrm{AUC}_{\mathrm{DCI}-\mathrm{IPG}}$ alone. Hence, we used this ratio in our analyses. Serum $\mathrm{AUC}_{\mathrm{DCI}-\mathrm{IPG}}$ $\mathrm{AUC}_{\mathrm{insulin}}$ ratios were not normally distributed and were therefore $\log$ transformed for statistical analyses and then reported back transformed in their original units.

Whole Body Insulin Sensitivity Index as described by Matsuda et al. ${ }^{18}$ was determined and compared within each group as well as across both groups at baseline and after treatment.

Comparisons between groups at baseline were made with a Student's two-tailed $t$ test. To assess within group effects from baseline to after treatment, a matched pairs two tailed $t$-test was performed. To assess the treatment effects between groups, the changes in each variable (after treatment minus baseline) were compared using a two-tailed $t$ test. A Pearson's correlation was used to assess the association between change in Matsuda index and change in bioactive DCI-IPG released per unit of insulin during OGTT. All results are reported as mean $\pm \mathrm{SE}$, except for graphics, in which means and $95 \%$ confidence intervals (CIs) are presented. $P<0.05$ was considered significant.

\section{Results}

\section{Baseline characteristics}

Women in the pioglitazone and placebo groups did not differ significantly with respect to age, BMI, waist to hip ratio, or serum-free testosterone concentrations (Table 1). They also did not differ in the relative bioactivity of DCI-IPG $\left(\mathrm{AUC}_{\mathrm{DCI}-\mathrm{IPG}}\right)$ curves during the OGTT or $\mathrm{AUC}_{\mathrm{DCI}-\mathrm{IPG}} /$ $\mathrm{AUC}_{\text {insulin }}$ ratios. While fasting insulin was not significantly different between the two groups at baseline, the $\mathrm{AUC}_{\mathrm{insulin}}$ during the OGTT was lower in the pioglitazone group than in the placebo group $(6649.39 \pm 236.67$ vs. $7595.57 \pm 269.11 \mu \mathrm{IU}$. $\mathrm{min} / \mathrm{mL}, P=0.01)$. None of the women exhibited impaired glucose tolerance.

\section{Anthropometric measurements at end of study}

As expected, weight and BMI increased significantly after treatment in the pioglitazone group only. In addition, the increase in weight and BMI was significantly higher than the placebo group $(P<0.0001)$. Despite increase in weight and $\mathrm{BMI}$, waist/hip ratio decreased slightly but significantly after treatment only within the pioglitazone group $(0.82 \pm 0.01$ to $0.80 \pm 0.01, P=0.001)$, and the decrease was statistically greater than that in the placebo group $(P=0.0094)$.

\section{Serum insulin and the bioactivity profiles of DCI-IPG after treatment}

After treatment, the serum fasting insulin decreased significantly from baseline to end of treatment only within the pioglitazone group $(15.04 \pm 1.23$ to $6.35 \pm 0.41 \mu \mathrm{IU} / \mathrm{mL} ; P<0.0001)$ and differed significantly from the change in placebo group $(P<0.0002$; Table 2$)$. Mean $\mathrm{AUC}_{\text {insulin }}$ decreased significantly from baseline within both the pioglitazone $(6649.39 \pm 236.67$ to $3634.58 \pm 152.74 \mu \mathrm{IU} \cdot \mathrm{min} / \mathrm{mL}, P<0.0001)$ and placebo groups $(7595.57 \pm 269.11$ to $6560.63 \pm 189.22 \mu \mathrm{IU} \cdot \mathrm{min} / \mathrm{mL}$, $P=0.0013$ ), but the decrease in the pioglitazone group was threefold greater than that in the placebo group, respectively $(-3014.8 \pm 273.78$ vs. $-1034.9 \pm 263.30 \mu \mathrm{IU} \cdot \mathrm{min} / \mathrm{mL}, P<0.001)$.

Whole body insulin sensitivity, as determined by the Matsuda index, increased significantly after treatment in the pioglitazone group $(3.36 \pm 0.18$ to $7.65 \pm 0.34, P<0.0001)$, whereas no significant change was observed in the placebo group ( $3.05 \pm 0.15$ to $3.35 \pm 0.18, P=0.10)$. The change in Matsuda index after treatment with pioglitazone differed significantly from that in the placebo group (4.29 $\pm 0.36 \mathrm{vs}$. $0.29 \pm 0.16$, respectively, $P<0.0001)$.

After treatment, mean $\mathrm{AUC}_{\mathrm{DCI}-\mathrm{IPG}}$ decreased from baseline within each group, but did not differ significantly between the two groups (Table 2). Nevertheless, the ratio of $\mathrm{AUC}_{\mathrm{DCI}-\mathrm{IPG}} / \mathrm{AUC}_{\mathrm{insulin}}$ increased from baseline to end-oftreatment in the pioglitazone group from $2.01 \% \pm 0.17 \%$ to $3.60 \% \pm 0.47 \% / \mu \mathrm{IU} \cdot \mathrm{min} / \mathrm{mL}(P<0.0001)$, whereas it $\mathrm{did}$ not change in the placebo group $(1.85 \% \pm 0.17 \%$ to $2.07 \% \pm$ $0.28 \% / \mu \mathrm{IU} \cdot \mathrm{min} / \mathrm{mL}, P=\mathrm{NS})$. The fold increase in the ratio of $\mathrm{AUC}_{\mathrm{DCI}-\mathrm{IPG}} / \mathrm{AUC}_{\text {insulin }}$ from baseline to end-of-treatment

Table 1. Baseline Clinical Characteristics and Serum Hormone Concentration of Women with PCOS

\begin{tabular}{|c|c|c|c|}
\hline Characteristic & Placebo $(\mathrm{n}=16)$ & Pioglitazone $(\mathrm{n}=16)$ & $\mathrm{P}(\mathrm{t}$-test $)$ \\
\hline Age (years) & $30.56(1.08)$ & $29.68(1.10)$ & 0.57 \\
\hline Weight (kg) & $68.90(1.10)$ & $68.31(0.65)$ & 0.66 \\
\hline $\mathrm{BMI}\left(\mathrm{kg} / \mathrm{m}^{2}\right)$ & $26.33(0.31)$ & $26.08(0.24)$ & 0.53 \\
\hline Waist/hip ratio & $0.80(0.01)$ & $0.82(0.01)$ & 0.13 \\
\hline Acne & $56.25 \%(9 / 16)$ & $56.25 \%(9 / 16)$ & 1.0 \\
\hline Serum-free testosterone (ng/dL) & $3.01(0.20)$ & $3.54(0.23)$ & 0.10 \\
\hline Fasting serum insulin $(\mu \mathrm{IU} / \mathrm{mL})$ & $15.91(1.32)$ & $15.04(1.23)$ & 0.63 \\
\hline $\operatorname{AUC}_{\text {insulin }}(\mu \mathrm{IU} \cdot \min / \mathrm{mL})$ & $7595.57(269.11)$ & $6649.39(236.67)$ & 0.01 \\
\hline HOMA-IR & $3.45(0.31)$ & $3.34(0.27)$ & 0.81 \\
\hline Matsuda index & $3.05(0.15)$ & $3.36(0.18)$ & 0.19 \\
\hline $\mathrm{AUC}_{\mathrm{DCI}-\mathrm{IPG}}(\% \mathrm{~min})$ & $14054.17(1373.1)$ & $13162.06(1240.6)$ & 0.63 \\
\hline Ratio of AUC ${ }_{\text {DCI-IPG }} / \mathrm{AUC}_{\text {insulin }}(\% / \mu \mathrm{IU} / \mathrm{mL})$ & $1.85(0.17)$ & $2.01(0.24)$ & 0.48 \\
\hline
\end{tabular}

Data are mean \pm SE. To convert values for total testosterone to nanomoles per liter, multiply by 0.0347 ; to convert values for free testosterone to picomoles per liter, multiply by 34.7 ; to convert insulin to picomoles per liter multiply by 7.2 . The normal ranges for ovulatory women are as follows: free testosterone $0.06-0.19 \mathrm{ng} / \mathrm{dL}$, insulin $5-20 \mu \mathrm{IU} / \mathrm{mL}$.

$P<0.05$ is level of significance. Significant figure highlighted in bold.

BMI, body mass index; IR, insulin resistance; PCOS, polycystic ovary syndrome. 
Table 2. Changes in Clinical Characteristics and Hormone Concentrations After the Administration of Placebo or Pioglitazone for 6 months in Women with PCOS

\begin{tabular}{|c|c|c|c|}
\hline Characteristic & Placebo & Pioglitazone & $\mathrm{P}(\mathrm{t}-$ test $)$ \\
\hline Weight (kg) & $+0.11(-0.42,0.65)$ & $1.71(1.35,2.09)$ & $<0.0001$ \\
\hline BMI $\left(\mathrm{kg} / \mathrm{m}^{2}\right)$ & $0.04(-0.17,0.25)$ & $0.66(0.51,0.80)$ & $<0.0001$ \\
\hline Waist/hip ratio & $0.00(-0.1,0.02)$ & $-0.02(-0.03,0.0)$ & 0.0094 \\
\hline Fasting serum insulin $(\mu \mathrm{IU} / \mathrm{mL})$ & $-0.24(-3.44,2.96)$ & $-8.69(-11.31,-6.08)$ & $<0.0002$ \\
\hline $\mathrm{AUC}_{\text {insulin }}(\mu \mathrm{IU} \cdot \mathrm{min} / \mathrm{mL})$ & $-1034.94(-1596,-474)$ & $-3014.81(-3598,-2431)$ & $<0.00018$ \\
\hline HOMA-IR & $-0.04(-0.78,0.70)$ & $-2.05(-2.62,-1.5)$ & $<0.00018$ \\
\hline Matsuda index & $0.29(-0.06,0.65)$ & $4.29(3.53,5.05)$ & $<0.0001$ \\
\hline $\mathrm{AUC}_{\mathrm{DCI}-\mathrm{IPG}}(\% \mathrm{~min})$ & $-699.45(-4729,3330.5)$ & $-412.22(-3174,2349.4)$ & 0.90 \\
\hline Ratio of AUC ${ }_{\text {DCI-IPG }} / \mathrm{AUC}_{\text {insulin }}(\% / \mu \mathrm{IU} / \mathrm{mL})$ & $1.21(0.88,1.54)$ & $1.85(1.50,2.20)$ & 0.0083 \\
\hline
\end{tabular}

Data are expressed as mean (95\% confidence interval). To convert insulin to picomoles per liter, multiply by 7.2 . The normal ranges for ovulatory women are insulin 5-20 $\mu \mathrm{IU} / \mathrm{mL}$.

$P<0.05$, level of significance. Significant figures highlighted in bold.

differed significantly between the two groups (pioglitazone group: $1.85 \pm 0.17$ vs. placebo group: $1.2 \pm 0.15, P=0.0083$, Fig. 1). There was no significant change in DCI-IPG during glucose tolerance test from 0 to 60 or $120 \mathrm{~min}$ in either group at baseline or after treatment.

When women from both groups were combined, there was a significant positive correlation $(r=0.47, P<0.01)$ between change in Matsuda index and change in DCI-IPG released per unit of insulin during OGTT (Fig. 2). However, this relationship was not significant when the groups were analyzed separately due to lack of statistical power. Nevertheless, there was a trend toward better correlation within pioglitazone group compared to the placebo group.

\section{Discussion}

In this randomized placebo-controlled study, we demonstrated that release of DCI-IPG per unit of insulin released

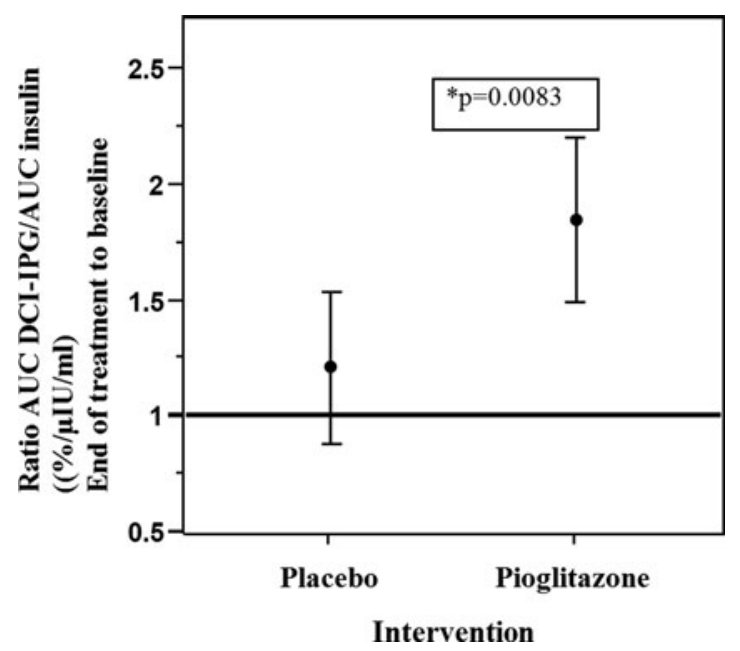

FIG. 1. Changes in the ratio of the $\mathrm{AUC}_{\text {insulin }} / \mathrm{AUC} \mathrm{C}_{\mathrm{DCI}-\mathrm{IPG}}$ curve during OGTT after the administration of pioglitazone or placebo for 6 months in women with PCOS. Values are expressed as mean change $\pm 95 \%$ confidence interval. The ratios were $\log$ transformed for statistical analysis and reported back transformed in original units so change represents the ratio of the result after treatment compared with baseline (no change is represented by a ratio of 1). OGTT, oral glucose tolerance test; PCOS, polycystic ovary syndrome. during an OGTT increased significantly by $85 \%$ after treatment with pioglitazone, but did not change after treatment with matched placebo. The increased release of DCIIPG per unit of insulin released in women treated with pioglitazone was accompanied by improved whole-body insulin sensitivity, as determined by the Matsuda Index, and reduced circulating insulin during an OGTT. We note that the increase in the ratio of $\mathrm{AUC}_{\mathrm{DCI}-\mathrm{IPG}}$ to $\mathrm{AUC}_{\mathrm{insulin}}$ during the OGTT in the pioglitazone group was due to a decrease in the $\mathrm{AUC}_{\text {insulin }}$ with no significant change in the $\mathrm{AUC}_{\mathrm{DCI}-\mathrm{IPG}}$. DCI-IPG is a second messenger of insulin action. To our knowledge, insulin is the only hormone or substance that has been reported to affect release of the DCI-IPG second messenger, as has been demonstrated both in vitro and in vivo in human beings. ${ }^{9}$ Consequently, we would expect a parallel drop in DCI-IPG with decreased insulin secretion. To the contrary, no decrease in DCI-IPG levels was observed in the study after treatment with pioglitazone, despite reduced insulinemia. Therefore, by enhancing insulinmediated release of DCI-IPG (i.e., a greater amount of DCIIPG released by target tissues per unit of insulin binding to

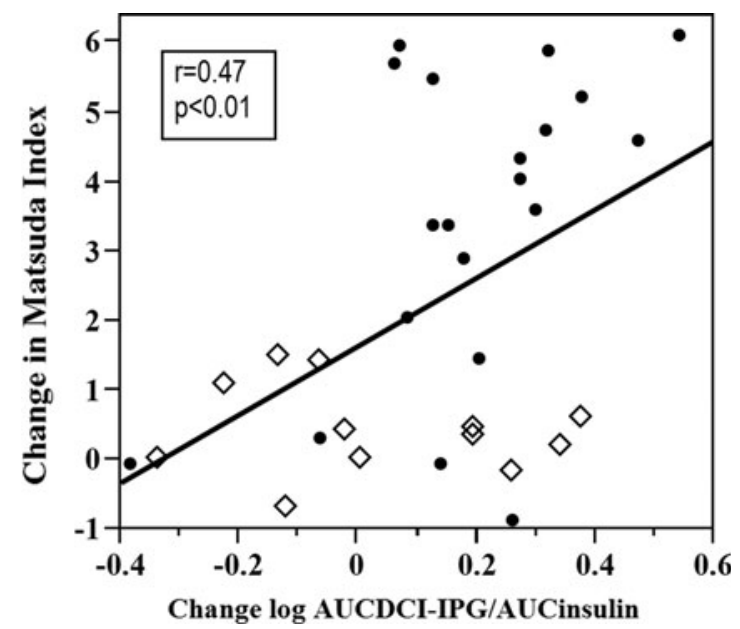

FIG. 2. Relationship between change in Matsuda index and change in release of the bioactive DCI-IPG messenger per unit of insulin released during OGTT in placebo $(\diamond)$ and pioglitazone $(\bullet)$ groups combined from baseline to end of study at 6 months. DCI-IPG, D-chiro-inositol-inositolphosphoglycan mediator. 
its receptor), pioglitazone reduced the amount of insulin that was required to handle the glucose load. Hence, the increase in the ratio of $\mathrm{AUC}_{\mathrm{DCI}-\mathrm{IPG}}$ to $\mathrm{AUC}_{\text {insulin }}$ during the OGTT after treatment with pioglitazone reflects an enhanced ability of insulin to affect release of DCI-IPG from the cell membrane of insulin target tissues. Specifically, since glucose tolerances were normal at baseline, there was no physiologic need for increased release of the DCI-IPG mediator. These results are qualitatively similar to those previously reported by Baillargeon et al. for metformin. ${ }^{6}$ Finally, we noted a significant positive association between change in Matsuda index and change in DCI-IPG released per unit of insulin, as has been demonstrated in our previous study with oral DCI. ${ }^{8}$

A major strength of the study is its randomized, doubleblind placebo-controlled design, which provides internal validity to the findings of improved DCI-IPG release per unit of insulin after administering pioglitazone. In addition, these observations are novel since there is no such study in thiazolidinediones, although results are similar to those previously reported for metformin. ${ }^{6}$ This provides support to and further reinforces the relevance of our findings of improved DCI-IPG per unit insulin after administering pioglitazone.

A weakness of the study is that specific mechanism to explain how pioglitazone improves DCI-IPG/insulin ratio remains to be elucidated; however, that was not the focus of the study. While speculative, in the absence of any other known mechanism for release of DCI-IPG, it is reasonable to suggest that improved insulin sensitivity through increased release of DCI-IPG per unit insulin may represent a common therapeutic pathway of action for several classes of insulin-sensitizing drugs. The mechanism by which pioglitazone improved the insulin-stimulated release of DCI-IPG remains unknown. Possibilities include enhanced intracellular signaling (i.e., coupling) between insulin binding and DCI-IPG release, increased intracellular production of the DCI-IPG messenger, amelioration of possible cell surface impediments to the release of DCI-IPG mediator, or a combination of such actions.

In conclusion, this study demonstrated that pioglitazone improves the insulin-stimulated release of the DCI-IPG second messenger of insulin action in women with PCOS. Combined with previous studies of metformin and DCI by our group and others, this study reinforces the contribution of DCI and its messenger in improving IR in women with PCOS.

\section{Acknowledgments}

The authors would like to thank Dr. Kai I. "Annie" Cheang, Ms. Maria Shulleeta, and Mr. Jamal T. Aldajaei for their invaluable assistance in conduct of the study (Jamal T. Aldajaei) and collecting (Maria Shulleeta) and analyzing data (Kai I "Annie" Cheang) toward preparation of the article. Supported by grants R01HD035629 (J.E.N.) and KL2TR000057 (A.G.).

\section{Authors' Contributions}

Dr. A.G. analyzed the data, drafted, edited, and prepared the final article. Dr. D.J. conducted the studies at Hospital de Caracas, Venezuela, reviewed and edited the article, and is currently working at the Wolfson Medical Center, Tel Aviv University, Israel. She had affiliation with both institutions at the time the study was conducted. Dr. J.E.N. designed the study and supervised conduct of the study, assays, and data analyses, as well as reviewed and edited the final article. All authors have read, approved the final version, and agreed to the publication of the article. Clinicaltrials.gov no.: NCT00868140

\section{Author Disclosure Statement}

Pioglitazone and matched placebo were provided by Takeda Pharmaceuticals. Takeda did not have any role in conduct of the study or preparation of the article. None of the authors has any other potential conflicts of interest.

\section{References}

1. Sirmans SM, Pate KA. Epidemiology, diagnosis, and management of polycystic ovary syndrome. Clin Epidemiol 2013;6:1-13.

2. Knochenhauer ES, Key TJ, Kahsar-Miller M, et al. Prevalence of the polycystic ovary syndrome in unselected black and white women of the southeastern United States: A prospective study. J Clin Endocrinol Metab 1998;83: 3078-3082.

3. Ciaraldi TP, el Roeiy A, Madar Z, et al. Cellular mechanisms of insulin resistance in polycystic ovarian syndrome. J Clin Endocrinol Metab 1992;75:577-583.

4. Nestler JE. Role of hyperinsulinemia in the pathogenesis of the polycystic ovary syndrome, and its clinical implications. Semin Reprod Endocrinol 1997;15:111-122.

5. Romero G, Larner J. Insulin mediators and the mechanism of insulin action. Adv Pharmacol 1993;24:21-50.

6. Baillargeon JP, Iuorno MJ, Jakubowicz DJ, et al. Metformin therapy increases insulin-stimulated release of a D-chiro-inositol-containing inositolphosphoglycan mediator in women with polycystic ovary syndrome. J Clin Endocrinol Metab 2004;89:242-249.

7. Asplin I, Galasko G, Larner J. Chiro-inositol deficiency and insulin resistance: A comparison of the chiro-inositol- and the myo-inositol-containing insulin mediators isolated from urine, hemodialysate, and muscle of control and type II diabetic subjects. Proc Natl Acad Sci USA 1993;90:59245928.

8. Cheang KI, Baillargeon JP, Essah PA, et al. Insulin-stimulated release of D-chiro-inositol-containing inositolphosphoglycan mediator correlates with insulin sensitivity in women with polycystic ovary syndrome. Metabolism 2008;57:1390-1397.

9. Larner J. Insulin-signaling mechanisms. Lessons from the old testament of glycogen metabolism and the new testament of molecular biology. Diabetes 1988;37:262-275.

10. Baillargeon JP, Iuorno MJ, Apridonidze $\mathrm{T}$, et al. Uncoupling between insulin and release of a D-chiro-inositolcontaining inositolphosphoglycan mediator of insulin action in obese women with polycystic ovary syndrome. Metab Syndr Relat Disord 2010;8:127-136.

11. Nestler JE, Jakubowicz DJ, Reamer P, et al. Ovulatory and metabolic effects of D-chiro-inositol in the polycystic ovary syndrome. N Engl J Med 1999;340:1314-1320.

12. Iuorno MJ, Jakubowicz DJ, Baillargeon JP, et al. Effects of D-chiro-inositol in lean women with the polycystic ovary syndrome. Endocr Pract 2002;8:417-423.

13. Gerli S, Mignosa M, Di Renzo GC. Effects of inositol on ovarian function and metabolic factors in women with PCOS: A randomized double blind placebo-controlled trial. Eur Rev Med Pharmacol Sci 2003;7:151-159.

14. Laganà AS, Barbaro L, Pizzo A. Evaluation of ovarian function and metabolic factors in women affected by 
polycystic ovary syndrome after treatment with D-chiroinositol. Arch Gynecol Obstet 2015;291:1181-1186.

15. Zawadzki J, Dunaif A. Diagnostic criteria for polycystic ovary syndrome: Towards a rational approach. In: Dunaif A, Given JR, Haseltine FP, Merriam GR (eds). Polycystic Ovary Syndrome: Current Issues in Endocrinology. Boston, MA: Blackwell Scientific; 1992: 377-384.

16. Romualdi D, Guido M, Ciampelli M, et al. Selective effects of pioglitazone on insulin and androgen abnormalities in normo- and hyperinsulinaemic obese patients with polycystic ovary syndrome. Hum Reprod 2003;18:1210-1218.

17. Shashkin PN, Shashkina EF, Fernqvist-Forbes E, et al. Insulin mediators in man: Effects of glucose ingestion and insulin resistance. Diabetologia 1997;40:557-563.
18. Matsuda M, DeFronzo RA. Insulin sensitivity indices obtained from oral glucose tolerance testing: Comparison with the euglycemic insulin clamp. Diabetes Care 1999;22: 1462-1470.

Address correspondence to: Anshu Gupta, MD Department of Pediatrics Virginia Commonwealth University PO 980140

Richmond, VA 23298

E-mail: anshu.gupta@vcuhealth.org 